\title{
Faster than the eye can see: blue cones respond to rapid flicker
}

\author{
Andrew Stockman, Donald I. A. MacLeod, and Stewart J. Lebrun \\ Department of Psychology, University of California, San Diego, La Jolla, California 92093-0109
}

Received August 4, 1992; revised manuscript received December 2, 1992; accepted December 4, 1992

\begin{abstract}
Flickering lights that are detected by the blue cones of the human visual system fuse to yield a steady sensation at much lower rates of flicker than do lights that are detected by the red or green cones. Yet, although bluecone-detected lights flickering at $30-40 \mathrm{~Hz}$ appear to be steady, they are still able to interact with red- or green-cone-detected flickering lights to produce clearly detectable beats in the form of an amplitude modulation of the red- or green-cone flicker. Thus the blue cones produce a viable high-frequency flicker signal, as do the red and green cones, but one that is normally lost before it reaches sensation. The temporal-frequency response for the blue-cone beat interaction is similar in shape to the temporal-frequency response for directly detected red- or green-cone flicker. When measured through the same pathway (which we identify as the luminance pathway, since it is able to transmit high-frequency flicker), the response of the blue cones seems to be as fast as that of the other cones.
\end{abstract}

\section{INTRODUCTION}

When our vision is restricted to signals from the bluesensitive cones, it is strikingly impoverished: we are limited not only in how much detail we can perceive $e^{1,2}$ but also in our ability to resolve rapid changes in intensity (i.e., flicker). The highest frequency to which flicker can be resolved is much lower for blue-cone-detected flicker than for red-or green-cone-detected flicker: upper limits of between 18 and $28 \mathrm{~Hz}$ have been reported for blue-cone flicker, ${ }^{3-5}$ whereas red- and green-cone flicker can be resolved up to $>50 \mathrm{~Hz}$ under comparable conditions. ${ }^{6,7}$

The temporal deficit of blue-cone-mediated vision could arise in the blue-cone photoreceptors themselves or in the postreceptoral pathways through which their signals travel. Under most conditions, blue-cone signals seem to be confined to the sluggish visual pathways with low-pass temporal-frequency responses that carry chromatic or color information and seem to have little or no access to the faster pathways that carry luminance or intensity information, ${ }^{8-16}$ which suggests that the deficit may arise postreceptorally. ${ }^{17,18}$ Recent evidence, however, indicates that the blue cones make a weak contribution to the faster, luminance pathway when intense long-wavelength light renders the other cones insensitive ${ }^{5,19,20}$ (see also Ref. 21). This blue-cone luminance input is characterized by a bandpass temporal-frequency response that extends to nearly $30 \mathrm{~Hz},{ }^{5,22}$ the ability to flicker-photometrically cancel flicker generated in the green or red cones, ${ }^{5}$ and the ability to provide information regarding the direction of the motion of moving gratings ${ }^{20}$ all of which distinguish the blue-cone luminance input from the blue-cone chromatic input (see Refs. 5, 20, and 23 for further experiments and discussion). By measuring the signals of the blue cones and those of the red and green cones through a common pathway, the luminance pathway, it should be possible to compare directly the temporal properties of the three cone types.

Here we also take advantage of a flicker interaction be- tween the blue cones and the other cones, which is observed as a beat phenomenon, to follow the temporal response of the blue cones to $40 \mathrm{~Hz}$. The beat phenomenon is illustrated in Fig. 1. At sufficiently high stimulus intensities, red- or green-cone-detected $40.5-\mathrm{Hz}$ flicker is clearly visible (top panels), but blue-cone-detected $40-\mathrm{Hz}$ flicker is not: by itself, it appears completely steady (bottom panels).

Even though the $40-\mathrm{Hz}$ blue-cone flicker cannot be resolved, it can interact with the $40.5-\mathrm{Hz}$ red- or green-cone flicker to produce a visible beat at the difference frequency of $0.5 \mathrm{~Hz}$ (middle panel). As illustrated in Fig. 1, the beat is perceived as a waxing and waning of the flicker amplitude once every $2 \mathrm{~s}$. For this beat to be seen at all, a $40-\mathrm{Hz}$ blue-cone signal must be present at the early stages of the visual system. Significantly, the beat is seen as an amplitude modulation of the red- or green-cone flicker signal at the low, difference frequency, not as a lowfrequency color or luminance change superimposed on a constant amount of $40.5-\mathrm{Hz}$ flicker. This suggests that the beat is produced by flicker cancellation and reinforcement within the pathway that transmits the $40.5 \mathrm{-Hz}$ redor green-cone-detected flicker.

In the experiments reported below, we measured the temporal-frequency response of the blue-cone beat interaction with red or green cones and also the conventional temporal-frequency response of each cone type for the direct detection of flicker. We found that, under comparable conditions, the frequency responses of the three cone types are very similar (see also Ref. 5, Fig. 9).

\section{METHODS}

\section{Apparatus}

The optical apparatus was a conventional five-channel, Maxwellian-view system with a 2-mm entrance pupil. The light source was a 900-W xenon arc lamp. The sizes of the test and field stimuli were defined by circular field 
STIMULUS

40.5- $\mathrm{Hz}$ red or green cone flicker
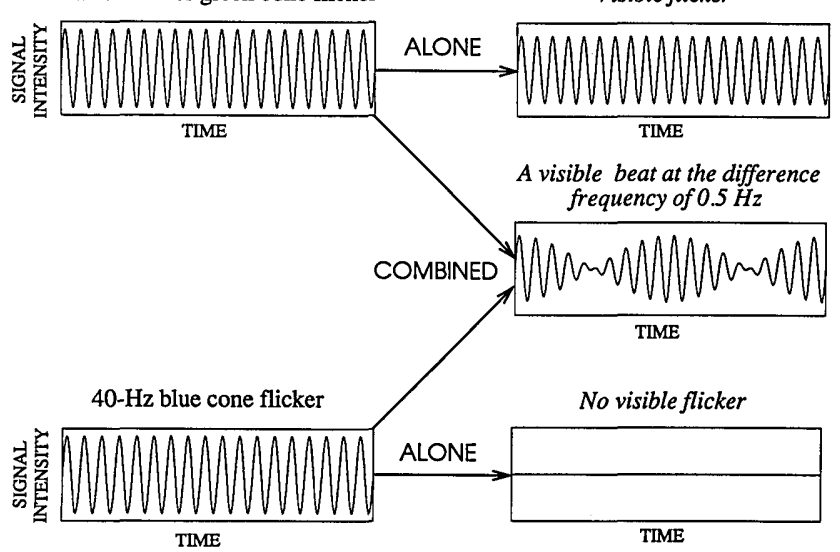

Fig. 1. Beat phenomenon. We can follow higher rates of flicker when our vision depends on signals from the red or green cones compared with when it is restricted to signals from the blue cones. At $40.5 \mathrm{~Hz}$ a red- or green-cone-detected stimulus can be seen to flicker (top panels), yet a $40-\mathrm{Hz}$ blue-cone-detected stimulus appears steady (bottom panels). When the invisibly flickering $40-\mathrm{Hz}$ blue-cone light is combined with the visibly flickering $40.5-\mathrm{Hz}$ red- or green-cone light, visible beats - a waxing and waning of the subjectively registered flicker amplitude - are seen at the difference frequency of $0.5 \mathrm{~Hz}$ as the two components come in phase and out of phase once every $2 \mathrm{~s}$ (middle panel). Figure is not shown to scale.

stops. Test and field wavelengths were selected with interference filters (Ealing) placed in the first collimated part of each beam. Infrared radiation was minimized by heat-absorbing glass (Oriel). The radiance of each beam could be varied by using fixed neutral-density filters (Inconel) or, under computer control, by using circular, variable neutral-density wedges (Inconel) mounted on stepping motors (Oriental Motor). Sinusoidal modulation was produced by pulse-width modulating fast, liquid-crystal light shutters (Displaytech) around a carrier frequency of $400 \mathrm{~Hz}$. Each shutter had rise and fall times of $<50 \mu \mathrm{s}$. The contrast of the shutter in the primary test channel measured in situ was 70:1 at $410 \mathrm{~nm}, 140: 1$ at $440 \mathrm{~nm}$, $230: 1$ at $470 \mathrm{~nm}$, and $>300: 1$ at $500 \mathrm{~nm}$ (and also at the longer target wavelengths that we used). The variability in contrast with wavelength has a minimal effect on the modulation depth produced by pulse-width modulation. The optical waveforms were monitored periodically by a Pin-10 photodiode (United Detector Technology) and an oscilloscope. The observer's head was stabilized by a dental wax impression rigidly mounted on adjustable cross slides that were taken from a milling machine.

\section{Stimuli}

To produce flicker that can be seen by only the blue cones, we took advantage of the fact that each cone type is most sensitive in a different region of the visible spectrum. We produced three circular fields of light on the subject's retina. The largest, an intense, steady orange background field $\{617 \mathrm{~nm}$, of 6-deg visual angle in diameter, producing $10^{11.6}$ quanta $\mathrm{s}^{-1} \mathrm{deg}^{-2}\left[5.08 \log _{10}\right.$ photopic trolands (ph. Td)] at the cornea\}, was included to reduce the sensitivities of the red and green cones, so that a small flickering violet test field superimposed on it $(440 \mathrm{~nm}$,
4 deg in diameter, $10^{9.3}$ quanta $\mathrm{s}^{-1} \mathrm{deg}^{-2}$ ) was detected by only the blue cones. A second small, orange flickering field $\left(609 \mathrm{~nm}, 4 \mathrm{deg}\right.$ in diameter, $10^{11.1}$ quanta $\mathrm{s}^{-1} \mathrm{deg}^{-2}$ ) was detected by the longer-wavelength cones but not by the blue cones. In AS, who is color normal, the 609-nm target would have been detected by his green and/or red cones, whereas in SJL, who is protanomalous (see below), the target would have been detected by his green and/or protanomalous red cones. The observers fixated the center of the fields.

\section{Procedures}

Each subject light adapted to the fields for at least $3 \mathrm{~min}$ before any data collection. Three types of measurement were made:

Flicker detection. To determine the temporal characteristics for directly detected blue-cone flicker, we varied the temporal frequency of the flickering blue-conedetected test light and measured the time-averaged fraction of the blue-cone light that must be flickered (the blue-cone modulation) for the flicker to be just detectable. The method of adjustment was used. Similar measurements were also made for red- and green-cone-detected flicker (see below).

Beat detection. To determine the temporal characteristics of the blue-cone contribution to the beat interaction illustrated in Fig. 1, we covaried the temporal frequency of the flickering components, keeping them $0.5 \mathrm{~Hz}$ apart in frequency and fixing the modulation of the red- or greencone flicker so that it was just above detection threshold (in fact, $0.2 \log _{10}$ unit above threshold), and measured the blue-cone modulation for the beat to be just detectable. Again, the method of adjustment was used.

Phase measurements. To determine the phase difference between the blue-cone-detected flicker and the redor green-cone-detected flicker, we used a variation of the beat procedure in which the two flickering components were always of the same frequency. To produce a temporal variation of the flicker that was similar in appearance to the beat phenomenon, both components were amplitude modulated at $0.5 \mathrm{~Hz}$. The subject's task was to adjust the phase difference between the blue-cone and the red- or green-cone flickering components to minimize the subjective flicker. The adjustment (away from the two components being physically out of phase) is then an estimate of the relative phase difference introduced by the visual system before the site(s) of flicker cancellation. In the experiment the subject was also able to vary the modulation of each component, and, by pressing a button, he could flip the relative phase of the two stimuli by $180 \mathrm{deg}$. Thus, at any point during the trial, the subject could compare the phase at which the flicker appeared least to an opposite phase at which the flicker appeared maximal. This helped to ensure that the minimum flicker setting was not erroneously obtained by adjusting the modulations of both components below threshold.

Except where noted, the data points are averaged from settings made on at least four separate runs.

\section{Calibration}

The relative spectral radiant power distributions of all light source and spectral filter combinations were measured in situ by using a calibrated EG\&G spectro- 
radiometer that had itself been calibrated against a reference mercury lamp and a reference light source. Measurements of the radiant fluxes of test and background fields were performed by using an EG\&G radiometer/photometer that had been cross-calibrated with a silicon photodiode (United Detector Technology) that was independently calibrated (by Optronics, Inc.) with a precision of $2 \%$ traceable to the National Bureau of Standards. The radiances are given below in quanta $\mathrm{s}^{-1} \mathrm{deg}^{-2}$; those for flickering lights are time-averaged radiances. Neutral-density filters, fixed and variable, were calibrated in situ for all test and field wavelengths used.

\section{Equivalent Quantum Catches}

We also compared the temporal-frequency response of the blue cones with the frequency responses of the other cones that were obtained under similar conditions of photoreceptor adaptation. Thus we chose test lights that give rise to similar quantal catches in the red or green cones as the 440-nm test light of the main experiment does in the blue cones. For SJL we used a single 4-deg-diameter, $609-\mathrm{nm}$ target, which would be detected by his anomalous cones and by his green cones. For AS, we used a single 4-deg-diameter, 668-nm target, which would be detected mainly, but not exclusively, by his red cones. Relative to the 440-nm target, the intense 617-nm background in the main experiment produced only a weak blue-cone stimulus [to the blue cones, the $10^{11.6}$ quanta s$~^{-1} \operatorname{deg}^{-2}\left(5.08 \log _{10} \mathrm{ph}\right.$. Td), 617-nm background is equivalent to a background of $\sim 10^{6.4}$ quanta $\mathrm{s}^{-1} \mathrm{deg}^{-2}$ at $440 \mathrm{~nm}$; see Ref. 5, p. 195). Since we also wanted to present the same spatial configuration to each cone type, the background was absent for the red- and green-cone temporal-sensitivity measurements. We calculated the relative quantum catches that were produced by the 440-nm target in the blue cones, the 609-nm target in the green cones (SJL), and the 668-nm target in the red cones (AS) by using the standard lens density estimate at $440 \mathrm{~nm}$ [Table II (2.4.6) of Ref. 24], by using standard red-and green-cone spectral sensitivities ${ }^{14}$ and assuming a macular density of 0.15 for a 4-deg-field at $440 \mathrm{~nm}$. We also assumed that the photopigment $\lambda_{\max }$ of the blue cones was $420 \mathrm{~nm}$ (see Ref. 25, Fig. 11) and that the quantum efficiency of the three cone types was equal at $\lambda_{\max }$. On this basis a $10^{10.1}$ quanta $\mathrm{s}^{-1} \mathrm{deg}^{-2} 668-\mathrm{nm}$ target, a $10^{9.6}$ quanta s${ }^{-1} \mathrm{deg}^{-2} 609-\mathrm{nm}$ target, and the $10^{9.3}$ quanta $\mathrm{s}^{-1} \mathrm{deg}^{-2} 440-\mathrm{nm}$ target of the main experiment give rise to similar quantum catches (per cone) in the red, green, and blue cones, respectively. We should emphasize that these equivalence calculations are at best approximate. For example, individual differences in prereceptoral filter density between subjects, or differences in quantum efficiency or cone-aperture size between cones, could lead to sizable differences in the actual quantum catches. The calculations for SJL were for his green cones. Assuming standard estimates of the $\lambda_{\max }$ of normal and anomalous cones, ${ }^{26}$ the quantum catch in his anomalous cones should be no more than two times that in his green cones. For reasons discussed below, the redcone temporal-frequency response for AS was actually measured by using a 668-nm target of $10^{10.96}$ quanta $\mathrm{s}^{-1} \mathrm{deg}^{-2}$. This target is approximately seven times more effective in stimulating the red cones than the standard $440-\mathrm{nm}$ target is in stimulating the blue cones.

\section{Subjects}

Two of the authors served as the observers in these experiments (AS and SJL). AS is emmetropic, and SJL is slightly myopic. SJL wore colorless correction lenses during the experiment. Both subjects are male. Color vision was tested by Rayleigh matches, the FarnsworthMunsell 100-hue test, and Ishihara plates. AS is trichromatic, and SJL is protanomalous. Both observers were fully informed about the general nature and possible consequences of the experiments, which posed no risk.

\section{RESULTS}

In the first experiment we measured the temporalfrequency response for the blue-cone beat interaction with the red or green cones. The results are shown in Fig. 2 as open circles for SJL (left panels) and AS (right panels), where they are plotted as modulation sensitivities, i.e., as the reciprocal of the threshold modulations of the bluecone-detected light that was required for beat detection.

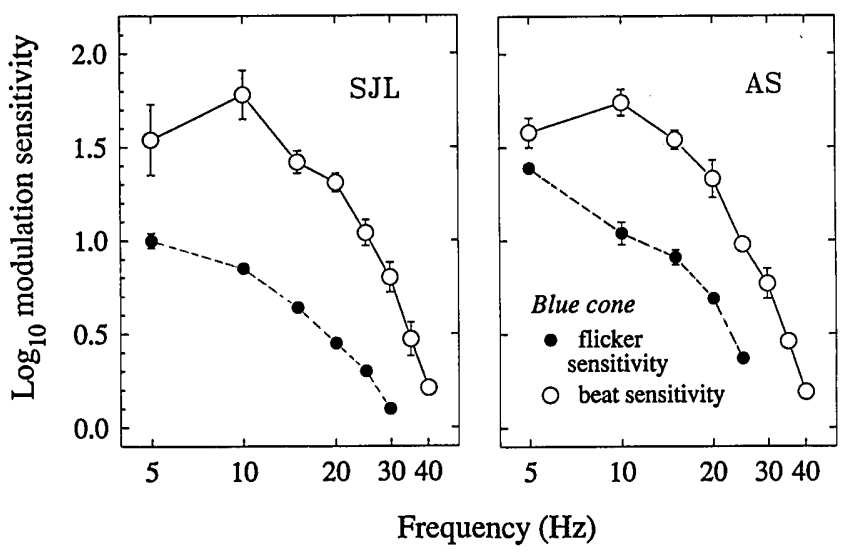

(a)

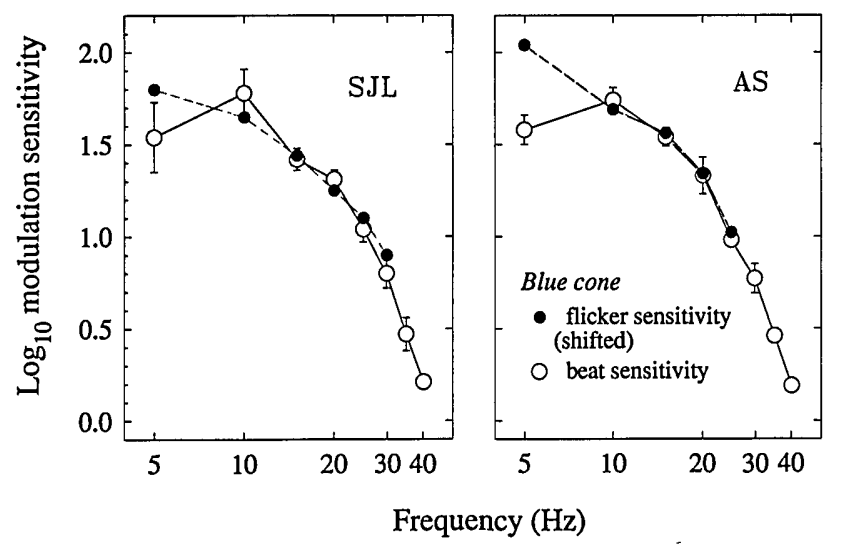

(b)

Fig. 2. Blue-cone temporal modulation sensitivities for subjects SJL (left panels) and AS (right panels) for detecting the beat interaction with a fixed-modulation red- or green-cone light (open circles) and for detecting flicker directly (filled circles). The error bars are \pm 1 standard error (SE). To show the agreement between the shapes of the blue-cone modulation sensitivities measured by using the two methods, in (b) the flicker-detection sensitivities have been shifted vertically to coincide with the beat-detection sensitivities. The blue-cone target was $440 \mathrm{~nm}$, $4 \mathrm{deg}$ in diameter, and $10^{9.3}$ quanta $\mathrm{s}^{-1} \mathrm{deg}^{-2}$, and the red- or green-cone target was $609 \mathrm{~nm}, 4 \mathrm{deg}$ in diameter, and $10^{11.1}$ quanta $\mathrm{s}^{-1} \mathrm{deg}^{-2}$. Both were presented on a 617-nm, 6-degdiameter, $10^{11.6}$-quanta $\mathrm{s}^{-1} \mathrm{deg}^{-2}$ background. 


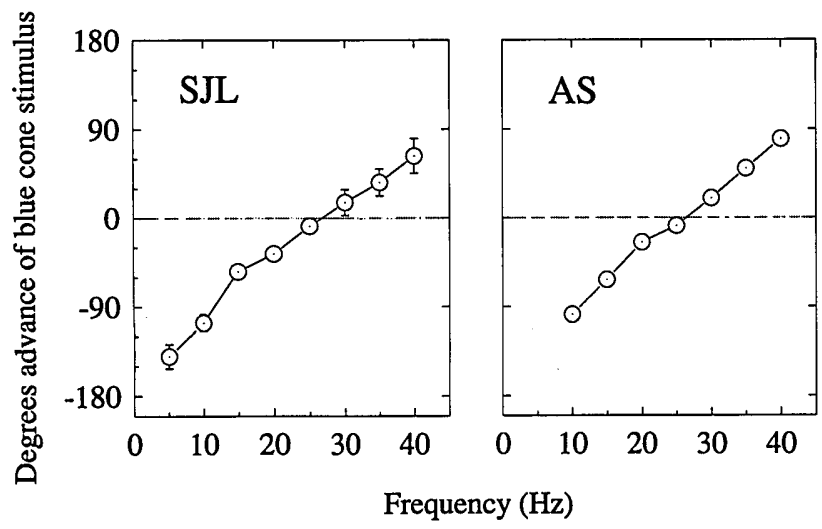

Fig. 3. Phase differences between blue-cone flicker and red- or green-cone flicker for SJL (left) and AS (right). The conditions are similar to those of Fig. 2. The error bars for SJL are $\pm 1 \mathrm{SE}$. (AS made only two sets of measurements.) The phase differences are the degrees advance of the blue-cone stimulus, relative to the out-of-phase red- or green-cone stimulus, that were needed to minimize the perception of flicker.

For comparison we also measured the blue-cone modulation sensitivities for directly detected flicker. Shown as filled circles in Fig. 2, these are comparable with results obtained under similar conditions by Stockman et al. ${ }^{5}$ In that paper we were able to show that this frequency response represents the envelope of the frequency responses of two S-cone pathways: (1) at low frequencies below $\sim 5 \mathrm{~Hz}$, a sluggish pathway with a low-pass temporalfrequency response that produces a chromatic percept; and (2) at higher frequencies, a faster pathway with a bandpass-frequency response that produces an achromatic percept that depends on a (vector) sum of signals from all three cone types (see Ref. 5). A similar distinction between a chromatic and a luminance S-cone pathway was made by Lee and Stromeyer ${ }^{20}$ on the basis of motion experiments.

Conventional blue-cone flicker, then, can be detected directly up to only $\sim 30 \mathrm{~Hz}$ (Fig. 2, filled circles). The sensitivity for detecting the interaction between blue-cone flicker and red- or green-cone flicker (Fig. 2, open circles) is approximately five times that for detecting blue-cone flicker itself. This improvement in sensitivity allows the blue-cone temporal response to be measured to $40 \mathrm{~Hz}$.

In the lower panels of Fig. 2 the blue-cone modulation sensitivities for flicker detection (filled circles) have been shifted upward to coincide with the sensitivities for beat detection (open circles). Actual shifts of 0.80 and $0.65 \log _{10}$ unit were required for SJL and AS, respectively. At $10 \mathrm{~Hz}$ and higher, where the conventional temporalfrequency response is determined by the faster, blue-cone luminance pathway, ${ }^{5}$ the shapes of the two functions are quite similar, which suggests that a common pathway, the luminance pathway, underlies both flicker and beat detection. The improvement in blue-cone sensitivity produced by the luminance-flicker interaction between the blue cones and the red or green cones is likely to be specific to the blue-cone luminance signal. Thus the discrepancy between the two functions at $5 \mathrm{~Hz}$ may arise because the direct detection of low-frequency flicker is mediated by low-pass chromatic pathways (see Ref. 5), whereas the detection of the beat depends on the bandpass luminance pathway. Alternatively, the shortfall in the beat sensitivity may exist because $5 \mathrm{~Hz}$ is too close to the beat frequency.

Figure 3 shows the phase difference between the bluecone-detected flicker and the red- or green-cone-detected flicker for SJL (left) and AS (right). Using the method described above, we were also able to measure the phase difference up to $40 \mathrm{~Hz}$. Consistent with other results obtained by using flicker cancellation or moving gratings, ${ }^{5,20,23,27}$ the phase difference tends toward $-180 \mathrm{deg}$ as the frequency tends toward $0 \mathrm{~Hz}$. This suggests that the S-cone input to luminance is actually inverted in sign. The large phase differences, coupled with the sign inversion, result in the blue-cone and red- or green-conedetected flicker's being synergistic near $26 \mathrm{~Hz}$ for both SJL and AS. If the phase differences shown in Fig. 3 were caused by an extra delay in the transmission of bluecone signals, the delay would be $\sim 19 \mathrm{~ms}$.

The beat sensitivity and phase measurements shown in Figs. 2 and 3 were made on an intense orange field to desensitize the red and green cones and thus ensure that the subthreshold violet flicker was detected by only the blue cones (see Fig. 5 below). As a result, the red and green cones are substantially more light adapted than the blue cones: in fact, the total time-averaged quantum catch in the blue cones is $\sim 500$ times less than that in the red cones and more than 100 times less than that in the green cones (see Equivalent Quantum Catches in the Methods section). Thus at least some of the large phase delay (relative to $-180 \mathrm{deg}$ ) seen in Fig. 3 is likely to be due to the fact that the blue cones (and any pathways whose sensitivity is determined primarily by the blue-cone signal) are less light adapted and therefore more sluggish than the other cones. Indeed, the temporal-frequency response for detecting the 609-nm flicker on the intense 617-nm field of the main experiment (not shown) is shallower than the blue-cone temporal-frequency response (shown in Fig. 2) by a factor of 0.25 (AS) or 0.4 (SJL) $\log _{10}$ unit by $40 \mathrm{~Hz}$. To compare the temporal-frequency response of the blue cones with those of the green (SJL) or red (AS) cones under similar conditions of adaptation, we chose test lights that give rise to similar quantal catches in each of the cone types. To present the same spatial configuration to each cone type, we omitted the background field (see Methods). The results are shown in Fig. 4.

The initial results for AS showed evidence of a bimodality that was consistent with chromatic flicker detection at low temporal frequencies - a conjecture that was supported by the finding that the same function measured under identical conditions in a deuteranomalous observer (DIAM) was unimodal. To avoid this problem, we measured the temporal-frequency response for AS at a slightly higher intensity, at which the low-frequency chromatic lobe is no longer evident.

The results in Fig. 4 demonstrate quite clearly that under comparable conditions of adaptation the blue-cone (open circles), red-cone (squares, AS) and green-cone (squares, SJL) temporal-frequency responses are very similar in shape. This is true even though the target used for AS is $\sim 7$ times more effective in stimulating the red cones than is the standard $440-\mathrm{nm}$ target in stimulating the blue cones (see above). 


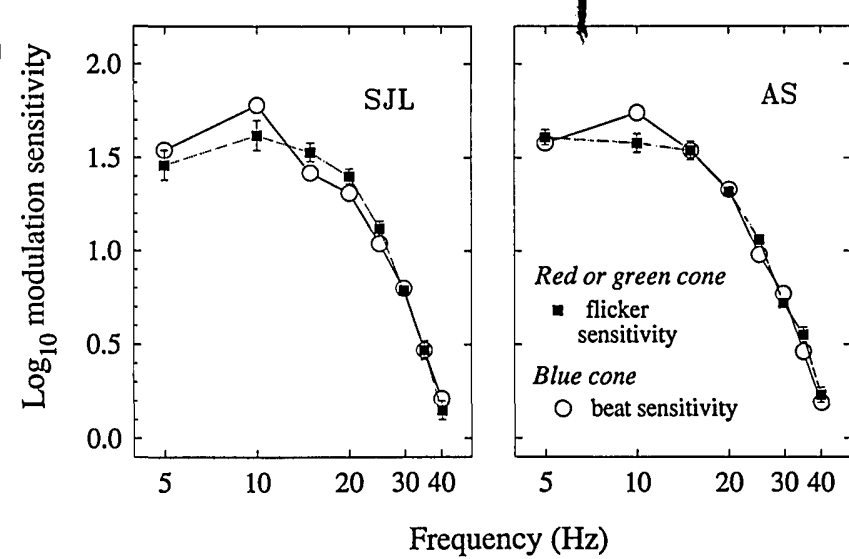

Fig. 4. Comparison between the blue-cone-modulation sensitivity for detecting the beat interaction (open circles, solid curve from Fig. 2) and the red- or green-cone-modulation sensitivities for detecting flicker directly (filled squares). The red- or greencone-modulation sensitivity functions have been shifted vertically downward ( $\times 0.39 \log _{10}$ unit for both subjects) to align with the blue-cone function. The measurements for SJL were made by using a single 4-deg-diameter, $10^{9.6}$ quanta $\mathrm{s}^{-1} \mathrm{deg}^{-2}, 609-\mathrm{nm}$ target, and those for AS were made by using a single $10^{10.96}$ quanta $\mathrm{s}^{-1} \mathrm{deg}^{-2}, 668-\mathrm{nm}$ field. The error bars are $\pm 1 \mathrm{SE}$.
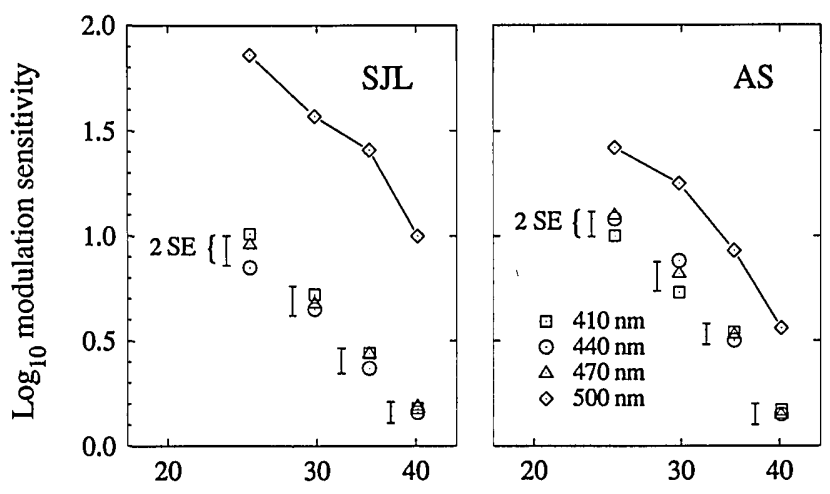

Frequency $(\mathrm{Hz})$

Fig. 5. Blue-cone-modulation sensitivities for subjects SJL (left) and AS (right) for detecting the beat interaction with a fixedmodulation red- or green-cone light measured by using 4-degdiameter targets of 410 (squares), 440 (circles), 470 (triangles), and 500 (diamonds) $\mathrm{nm}$ that were equated in their effects on the blue cones. The radiances of the targets were $10^{9.70}(410), 10^{9.30}$ $(440), 10^{9.50}(470)$, and $10^{10.23}(500)$ quanta $\mathrm{s}^{-1} \mathrm{deg}^{-2}$ for SJL and $10^{9.60}(410), 10^{9.30}(440), 10^{9.48}(470)$, and $10^{10.22}$ (500) quanta $\mathrm{s}^{-1} \mathrm{deg}^{-2}$ for AS (see text). Measurements were made on the standard 6-deg-diameter $10^{11.6}$ quanta $\mathrm{s}^{-1} \mathrm{deg}^{-2}, 617-\mathrm{nm}$ background field. The error bars are $\pm 2 \mathrm{SE}$ averaged across wavelength.

Our experiments depend crucially on the assumption that the $440-\mathrm{nm}$ subthreshold flicker is detected solely by the blue cones. To check that this was indeed the case, we repeated the beat experiment shown in Fig. 2 by using targets of 410,470 , and $500 \mathrm{~nm}$ that were equated in their effects on the blue cones to the standard 440-nm target used in the main experiment. To equate the targets for the blue cones, we measured the blue-cone spectral sensitivity of our two subjects by finding the amplitudes of single, 410-, 440-, 470-, or 500-nm, 4-deg-diameter, targets, sinusoidally flickering at 2,5 , and $10 \mathrm{~Hz}$ (SJL) or 2 and $5 \mathrm{~Hz}$ (AS), at which the flicker was just at threshold. As before, the targets were presented on the intense orange 617-nm background. Since the blue cones are relatively insensitive to high-frequency flicker (see, e.g., Ref. 13), we used low-frequency flicker as a further guarantee of blue-cone isolation. Since we found no frequencydependent differences in spectral sensitivity between 2 and $10 \mathrm{~Hz}$ we averaged the results across frequency. The average $\log _{10}$ quantal spectral sensitivities, relative to a peak of 0 at $440 \mathrm{~nm}$, were $-0.40,-0.20$, and -0.93 at 410 , 470 , and $500 \mathrm{~nm}$, respectively, for SJL and $-0.30,-0.18$, and -0.92 for AS. The radiances of the 410-, 470-, and 500-nm targets were adjusted according to these spectral sensitivities to equate the four targets for their effects on the blue cones.

Figure 5 shows the temporal-frequency response for the blue-cone beat interaction that was measured between 25 and $40 \mathrm{~Hz}$ at 410 (squares), 440 (circles), 470 (triangles), and 500 (diamonds) $\mathrm{nm}$. As before, the suprathreshold component, flickering at a slightly different frequency, was $609 \mathrm{~nm}$. The bars to the left of the lower points are twice the mean SE averaged across target wavelength.

The data points at 410,440 , and $470 \mathrm{~nm}$ lie close to each other at all frequencies. Only at $500 \mathrm{~nm}$ is there any evidence for either subject of flicker detection by a photoreceptor type other than the blue cones. When compared with the orange background, these targets provide a weak stimulus to the longer-wavelength cones. If the green cones had detected the subthreshold flickering component in this experiment, the 410-, 440-, 470-, and 500$\mathrm{nm}$ thresholds would have been separated by steps of $\sim 0.25,0.6$, and $1.1 \log _{10}$ unit (estimated from the Smith and Pokorny green- and blue-cone spectral sensitivities). ${ }^{14}$ Clearly, this is not the case. The only large separations that are found are between the 470- and 500-nm modulation sensitivities, and those are only 0.9 and $0.4 \log _{10}$ unit for SJL and AS, respectively. Thus blue-cone isolation at $440 \mathrm{~nm}$, the target used in the main experiment, is excellent for both subjects.

\section{DISCUSSION}

The similarity between the blue- and red- or green-cone temporal-frequency responses (Fig. 4) and, further, the fact that the blue- and red- or green-cone signals can interact to produce beats imply that the signals are being transmitted through a common pathway. If the pathways for blue-cone and other cone signals were different, the mutual cancellation implicit in the beat phenomenon would not be possible. Since this common pathway can transmit $40.5-\mathrm{Hz}$ flicker, we identify it as the brisk, luminance pathway (see also below). Through this pathway the blue cones are as fast as the other cones. This suggests that the temporal deficit that is normally associated with bluecone-mediated vision is postreceptoral in origin.

We do not have phase data for blue-, green-, and redcone-detected flicker under equivalent conditions of adaptation, since blue-cone isolation cannot be ensured at the lower levels of red- and green-cone adaptation that were used to obtain the data shown in Fig. 4. It is tempting to conclude that the phase differences in Fig. 3, which (relative to $-180 \mathrm{deg}$ ) are consistent with a latency difference of $19 \mathrm{~ms}$, can be entirely attributed to the large differences in adaptation level between the blue cones and the red and green cones under the conditions of Fig. 3. Since the quantum catch in the green cones was $\sim 100$ times 
that in the blue cones (see above), the change in latency with adaptation level would have to be of the order of $10 \mathrm{~ms}$ per decade change in intensity for the blue- and green-cone signals to be in phase (or actually out of phase, since the blue-cone signal is inverted in sign) at comparable adaptation levels. And indeed, changes in latency with adaptation of that order have been reported in the literature (for example, Refs. 28 and 29). Although the phase differences in Fig. 3 are consistent with a simple latency difference, since they are nearly linear with frequency, they could also be consistent with a change in persistence (e.g., resulting from adaptational changes in the time constants of a series of linear temporal filters with cutoff frequencies somewhat higher than $40 \mathrm{~Hz}$ ). Some change in persistence is suggested by the fact that red-or green-cone temporal-frequency responses measured on the intense orange background (not shown) are shallower than those obtained under the equivalent quantum catch conditions of Fig. 4.

The opposition of the blue-cone signal to the red- and green-cone signals indicated by the phase differences in Fig. 3 might be taken to suggest that we are dealing here not with the luminance pathway but with a yellow-blue chromatic pathway. Yet, two properties of the highfrequency blue-cone signal argue against such an interpretation. First, flicker that is detected by the faster blue-cone pathway produces an achromatic percept that can be flicker photometrically canceled by red- or greencone flicker; it does not produce, as might be expected of a yellow-blue chromatic pathway, a chromatic percept. Second, the temporal-frequency response of the highfrequency blue-cone signal extends to $40 \mathrm{~Hz}$ with no more loss of sensitivity than the red- or green-cone signal (see Fig. 4), well beyond the temporal-frequency response of any psychophysically defined chromatic pathway (see, e.g., Refs. 11 and 30). Without some fundamental change in the operational definitions of the luminance and chromatic pathways, it must be concluded that the high-frequency blue-cone signal, although somewhat atypical, is a luminance signal.

The improvement in sensitivity that is provided by the beat method is consistent with a model in which the combined luminance flicker signal remains undetectable until it exceeds a relatively high threshold level. Alone, the weak blue-cone flicker signal is unable to exceed threshold, but, combined with the slightly mistuned, justsuprathreshold red- or green-cone flicker, it produces beats - small, discriminable variations in the amplitude of the weak flicker signal (Fig. 1). The beats themselves are not the result of nonlinear demodulation but may be generated by a purely linear combination of signals from the different types of cones. The threshold nonlinearity must be invoked only to account for the ability of a subthreshold stimulus to produce visible beats. The threshold nonlinearity is not specific to the blue-cone luminance signal; it is also revealed in experiments in which subthreshold redor green-cone flicker is combined with slightly mistuned suprathreshold red- or green-cone flicker. ${ }^{19}$

To conclude, we have demonstrated that the temporalfrequency response of the human blue cones is comparable with that of the red or green cones. Our findings thus concur with physiological recordings from isolated primate photoreceptors, which suggests that the light-induced flash response of blue-cone outer segments is not very different in waveform from those of green- or red-cone outer segments. ${ }^{31,32}$

\section{ACKNOWLEDGMENTS}

This research was supported by National Science Foundation grants BNS 88-12401 and IBN 92-10046. Additional support was provided by National Institutes of Health grant EY01711. We thank Sabine Apitz and Harvey Smallman for helpful comments.

\section{REFERENCES}

1. W. S. Stiles, "Incremental thresholds and the mechanisms of colour vision," Doc. Ophthalmol. 3, 138-163 (1949).

2. G. S. Brindley, "The summation areas of human colourreceptive mechanisms at increment threshold," J. Physiol. (London) 124, 400-408 (1954).

3. G. S. Brindley, J. J. Du Croz, and W. A. H. Rushton, "The flicker fusion frequency of the blue-sensitive mechanism of colour vision," J. Physiol. (London) 183, 497-500 (1966).

4. L. E. Marks and M. H. Bornstein, "Spectral sensitivity by constant CFF: effect of chromatic adaptation," J. Opt. Soc. Am. 63, 220-226 (1973).

5. A. Stockman, D. I. A. MacLeod, and D. D. DePriest, "The temporal properties of the human short-wave photoreceptors and their associated pathways," Vision Res. 31, 189-208 (1991).

6. S. Hecht and E. L. Smith, "Intermittent stimulation by light. VI. Area and the relation between critical frequency and intensity," J. Gen. Physiol. 19, 979-989 (1936).

7. C. W. Tyler and R. D. Hamer, "Analysis of visual modulation sensitivity. IV. Validity of the Ferry-Porter law," J. Opt. Soc. Am. A 7, 743-758 (1990).

8. E. Schrödinger, "Über das Verhältnis der Vierfarben zur Dreifarbentheorie," Sitzungsber. Akad. Wiss. Wien Math. Naturwiss. Kl. Abt. 2A 134, 471 (1925).

9. R. Luther, "Aus dem Gebiet der Farbreizmetrik," Z. Tech. Phys. 8, 540-558 (1927).

10. G. L. Walls, "A branched-pathway schema for the color-vision system and some of the evidence for it," Am. J. Ophthalmol. 39, 8-23 (1955).

11. H. De Lange, "Research into the dynamic nature of the human fovea-cortex systems with intermittent and modulated light. II. Phase shift in brightness and delay in color perception," J. Opt. Soc. Am. 48, 784-789 (1958).

12. S. L. Guth, J. V. Alexander, J. I. Chumbly, C. B. Gillman, and M. M. Patterson, "Factors affecting luminance additivity at threshold," Vision Res. 8, 913-928 (1968).

13. D. H. Kelly, "Spatio-temporal frequency characteristics of color-vision mechanisms," J. Opt. Soc. Am. 64, 983-990 (1974).

14. V. C. Smith and J. Pokorny, "Spectral sensitivity of the foveal cone photopigments between 400 and $500 \mathrm{~nm}$," Vision Res. 15, 161-171 (1975).

15. J. J. Wisowaty and R. M. Boynton, "Temporal modulation sensitivity of the blue mechanism: measurements made without chromatic adaptation," Vision Res. 20, 895-909 (1980).

16. A. Eisner and D. I. A. MacLeod, "Blue sensitive cones do not contribute to luminance," J. Opt. Soc. Am. 70, 121-123 (1980).

17. J. D. Mollon and J. Krauskopf, "Reaction time as a measure of the temporal response properties of individual colour mechanisms," Vision Res. 13, 27-40 (1973).

18. V. C. Smith, R. W. Bowen, and J. Pokorny, "Threshold temporal integration of chromatic stimuli," Vision Res. 24, 653660 (1984).

19. A. Stockman and D. I. A. MacLeod, "Visible beats from invisible flickering lights: evidence that blue-sensitive cones respond to rapid flicker," Invest. Ophthalmol. Vis. Sci. Suppl. 27, 71 (1986). 
20. J. Lee and C. F. Stromeyer III, "Contribution of human shortwave cones to luminance and motion detection," J. Physiol. (London) 413, 563-593 (1989).

21. B. Drum, "Short-wavelength cones contribute to achromatic sensitivity," Vision Res. 23, 1433-1439 (1983).

22. D. G. Green, "Sinusoidal flicker characteristics of the coloursensitive mechanisms of the eye," Vision Res. 9, 591-601 (1969).

23. C. F. Stromeyer III, R. T. Eskew, R. E. Kronauer, and L. Spillman, "Temporal phase response of the short-wave cone signal for color and luminance," Vision Res. 31, 787-803 (1991).

24. G. Wyszecki and W. S. Stiles, Color Science, 2nd ed. (Wiley, New York, 1982).

25. A. Stockman, D. I. A. MacLeod, and N. E. Johnson, "Spectral sensitivities of the middle- and long-wavelength sensitive cones," J. Opt. Soc. Am. A (to be published).

26. J. Pokorny, V. C. Smith, and I. Katz, "Derivation of the photopigment absorption spectra in anomalous trichromats," J. Opt. Soc. Am. 63, 232-237 (1973).
27. A. Stockman and D. I. A. MacLeod, "An inverted S-cone input to the luminance channel: evidence for two processes in S-cone flicker detection," Invest. Ophthalmol. Vis. Sci. Suppl. 28, 92 (1987).

28. M. Alpern, "A note on visual latency," Psychol. Rev. 75, 260264 (1968).

29. R. Vuorinen, Latency Determination and Temporal Filtering of Retinal Signals in Photopic Vision (Suomalainen Tiedeakatemia, Helsinki, 1989).

30. J. J. Wisowaty, "Estimates for the temporal response characteristics of chromatic pathways," J. Opt. Soc. Am. 71, 970977 (1981).

31. D. A. Baylor, B. J. Nunn, and J. L. Schnapf, "Spectral sensitivity of the cones of the monkey Macaca fascicularis," J. Physiol. (London) 390, 145-160 (1987).

32. J. L. Schnapf, B. J. Nunn, M. Meister, and D. A. Baylor, "Visual transduction in cones of the monkey Macaca fascicularis," J. Physiol. (London) 427, 681-713 (1990). 